\title{
Bilan de la gestion intégrée des ressources et du territoire au Québec
}

\author{
par Anne Bernard ${ }^{1}{ }^{*}$, Nancy Gélinas ${ }^{1}$ et Louis Bélanger ${ }^{1}$
}

\begin{abstract}
RÉSUMÉ
Le concept de gestion intégrée des ressources et du territoire (GIRT) apparaît dans le premier article de la Loi sur l'aménagement durable des forêts du Québec. La GIRT reste néanmoins un concept très abstrait en raison des nombreuses interprétations qui peuvent en être faites. Afin de faire un bilan de la mise en œuvre de la GIRT au Québec, différents résultats ont été analysés soit les constats faits lors d'un colloque intitulé Où en sommes-nous 30 ans après le colloque "L'utilisation polyvalente de la forêt, une utopie? » qui s'est tenu le 10 mai 2018 dans le cadre de la $86^{\mathrm{e}}$ édition de l'ACFAS et les résultats d'entrevues avec des experts impliqués dans la mise en œuvre de la GIRT. Bien que plusieurs changements se soient opérés au cours des trente dernières années, les résultats démontrent que des mises à jour sont toujours nécessaires pour atteindre les objectifs fixés dans le régime forestier de 2013. Ces changements devraient principalement s'opérer par une prise en compte des acteurs et des communautés ancrées sur le territoire, et ce, grâce à des approches de gouvernance participative.
\end{abstract}

Mots-clés : Gestion intégrée, polyvalence, régime forestier, Québec, participation citoyenne.

\section{ABSTRACT}

Integrated land and resource management (ILRM) concept is included in the first article of the Sustainable Forest Development Act of Québec. Nevertheless, ILRM remains a very abstract concept because of the many interpretations that can be made. In order to assess ILRM implementation in the Québec province, different results were analyzed, namely the findings made during the conference entitled Where are we now 30 years after the conference "Multipurpose use of the forest, a utopia?" which was held on May 10, 2018 as part of the $86^{\text {th }}$ edition of ACFAS and the results of interviews with experts involved in the ILRM implementation. Although several changes have taken place over the past 30 years, the results show that updates are still necessary to reach the objectives set in the 2013 forest regime. These changes should mainly consider the stakeholders and communities rooted in the territory, and that, through adapted governance tools.

Keywords: Integrated management, multipurpose, forest regime, Québec, citizen participation.

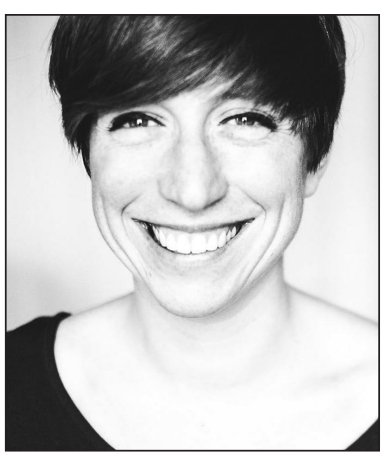

Anne Bernard

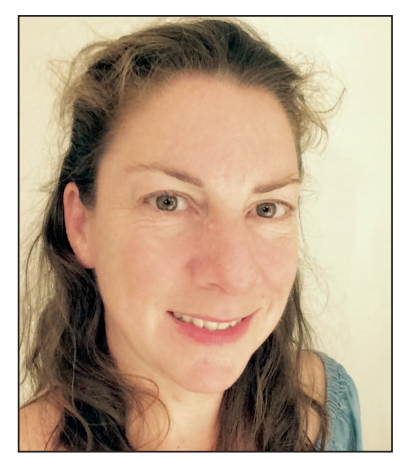

Nancy Gélinas

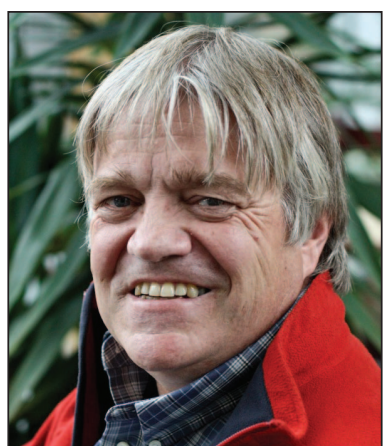

Louis Bélanger

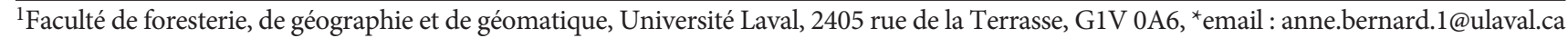




\section{Introduction}

Au Québec, la gestion intégrée des ressources et du territoire (GIRT) est l'une des approches mises en œuvre pour atteindre les objectifs d'aménagement forestier durable. Cependant, ce concept a évolué au fil des années, et ce, en fonction des outils réglementaires mis en place par le gouvernement. Dans les années 80, il était question de l'utilisation polyvalente de la forêt et aujourd'hui, il est question de la GIRT. À ce sujet, en 1986, année de l'adoption d'une nouvelle Loi sur les forêts, se tenait un colloque de l'ACFAS ${ }^{1}$ intitulé L'utilisation polyvalente de la forêt, une utopie? Le titre révélateur de ce colloque laissait présager un besoin de l'époque de prendre en compte les différents acteurs de la forêt pour mettre en ouvre l'utilisation polyvalente de la forêt. C'est pourquoi près de 30 ans plus tard, un colloque intitulé Où en sommes-nous 30 ans après le colloque "L'utilisation polyvalente de la forêt, une utopie? » a été organisé afin de dresser un bilan du concept. Afin d'avoir des perspectives variées, le colloque s'est organisé selon quatre thématiques : la GIRT perçue par le milieu faunique, la GIRT dans une perspective sociale, la GIRT dans le milieu industriel et finalement les perspectives d'amélioration continue pour la mise en ouvre de la GIRT. De plus, au fil des présentations traitant des thématiques fauniques, sociales et industrielles, trois aspects devaient être abordés soit la perspective du passé, celle du présent et finalement celle du futur. Pour l'occasion, des chercheurs, différents professionnels aux expertises variées et des étudiants ont participé à cette conférence.

L'objectif du présent article est de faire un bilan de la mise en œuvre du concept de GIRT depuis les trente dernières années. Pour ce faire, l'article se divise en quatre sections. La première présente une évolution du concept la GIRT. La seconde section présente les éléments clés qui distinguent le concept de 1986 de celui mis en œuvre aujourd'hui, à partir des présentations faites par les experts lors du colloque. La troisième section présente la perspective des experts responsables de la mise en œuvre de la GIRT dans sept régions administratives du Québec (Bernard et Gélinas, 2020). À la lumière des sections précédentes, la dernière section propose des pistes de réflexion pour l'adaptation du concept de la GIRT au contexte forestier actuel et futur.

\section{Évolution du concept de la gestion intégrée}

À l'époque où l'utilisation polyvalente des forêts commence à être présente sur la scène publique et politique, le Québec vit des changements importants quant à la gestion de ses ressources forestières. En 1970, le gouvernement du Québec débute un inventaire exhaustif de ses ressources qui est depuis actualisé tous les 10 ans; il s'agit de l'inventaire forestier mené par la direction de l'inventaire forestier (MFFP 2020a). À la même époque, la population tente de se réapproprier les territoires fauniques alors réservés à une élite en partie étrangère. En 1976, le gouvernement met sur pied l'Opération gestion faune afin de repenser la gestion de la faune par une utilisation plus démocratique des territoires pour la pratique des activités de chasse et de pêche en abolissant les clubs privés. C'est lors de la déclaration ministérielle du 22 décembre 1977 que le ministre Yves-L. Duhaime, alors ministre responsable du Tou-

\footnotetext{
$\overline{{ }^{1} \text { Association canadienne-française pour l'avancement des sciences }}$
}

risme, de la Chasse et de la Pêche, annonce qu'aucun bail à droits exclusifs ne sera renouvelé (Fédération québécoise des gestionnaires de zecs 2003). On assiste alors au « déclubage " du Québec qui permet la création des zones d'exploitation contrôlée (zec) que l'on connait aujourd'hui. Suite à de nombreux échanges avec les groupes d'acteurs impliqués dans le milieu faunique, des associations sont mises en place pour assurer la gestion de ces territoires afin de répondre à quatre objectifs principaux soit : faciliter l'accessibilité des citoyens, assurer la participation des utilisateurs (membres), permettre la conservation de la faune et assurer un autofinancement des activités ayant cours dans les territoires de zec (Fédération québécoise des gestionnaires de zecs 2003). Tous les citoyens québécois qui le désirent peuvent devenir membres d'une ou de plusieurs zecs. Encore aujourd'hui, la mission des zecs reste la même à quelques différences près puisqu'elles ont pour objectif d'aménager, d'exploiter et de conserver la faune tout en facilitant l'accès aux territoires à la société québécoise (Réseau Zec 2020). C'est aussi en 1977 que le gouvernement du Québec se dote de la Loi sur les parcs afin d'établir des parcs de conservation et de récréation restreignant l'exploitation des ressources naturelles sur certaines parties du territoire (Sépaq 2019).

Dans ce contexte de mise à jour des règlements et des lois, la prise en compte d'un plus grand nombre d'utilisateurs devient un aspect important de la gestion du territoire forestier. Les spécialistes de l'époque voulaient que le territoire forestier soit aménagé dans une perspective d'utilisation polyvalente. Par ailleurs, à la lecture des actes du colloque de 1986, les acteurs tentaient de faire reconnaitre leur légitimité auprès du gouvernement. À l'aube du régime forestier de 1986, les experts désirent mettre en place un projet de société pour le Québec, en misant sur une meilleure intégration des objectifs communs sur le territoire forestier (Regroupement pour un Québec Vert 1988). Encore aujourd'hui, cet objectif d'intégration des besoins et des valeurs des autres utilisateurs du territoire demeure une préoccupation centrale.

En 1987, le ministre du Loisir, de la Chasse et de la Pêche dépose un mémoire au comité ministériel permanent de l'aménagement, du développement régional et de l'environnement (COMPADRE) dans lequel il expose sa volonté de mettre en place un cadre de référence sur la gestion intégrée des ressources (GIR) dans les réserves fauniques. En 1989, cette initiative est aussi appuyée par le ministère de l'Énergie et des Ressources. À l'époque, les deux ministères proposent de mettre en place des projets-pilotes dans deux réserves fauniques afin de développer une méthode claire qui met en œuvre des objectifs sectoriels pour la gestion commune des ressources. Voici les étapes d'élaboration de la démarche, tirées du rapport final du projet GIR (Ministère des Ressources naturelles, Ministère de l'Environnement et de la Faune 1998) :

- Détermination des objectifs sectoriels pour chacune des ressources

- Élaboration de plans d'action sectoriels axés sur la poursuite de ces objectifs

- Intégration des préoccupations sectorielles, en vue de l'élaboration de scénarios d'aménagement intégré

- Évaluation des scénarios selon une approche multicritère

- Choix du scénario d'aménagement qui offre le meilleur compromis

À la lecture de cette démarche, on saisit l'ampleur du tra- 
vail accompli par les partenaires ayant participé à ce projetpilote. En plus de développer une réelle approche d'intégration des objectifs sectoriels, ces expérimentations ont aussi permis de raffiner les connaissances écologiques à l'étude. Plusieurs outils sont développés ce qui permet, entre autres, d'élaborer des indices de qualité d'habitat pour diverses espèces fauniques, de délimiter des aires équivalentes de coupe dans les bassins versants afin d'améliorer la protection de la ressource hydrique et de faire des analyses de retombées socioéconomiques pour mieux comprendre les impacts des opérations forestières. Cependant, l'élément central qui ressort de cette démarche est la proposition d'une analyse multicritère qui doit faciliter la prise de décision dans un contexte territorial complexe (Ministère des Ressources naturelles 1997). Il s'agit d'une méthode qui vise à identifier les critères et les indicateurs prioritaires pour les utilisateurs pour ensuite faire un choix d'aménagement qui répond aux attentes des utilisateurs et des gestionnaires.

Plusieurs recommandations découlent de cette expérience (Ministère des Ressources naturelles (MRN) et Ministère de l'Environnement et de la Faune (MEF) 1998) Parmi celles-ci, trois représentent la position gouvernementale de l'époque et témoignent du chemin qu'il reste encore à parcourir :

"Recommandation 6: Qu'à compter de la prochaine génération du plan de gestion des réserves fauniques ou du plan quinquennal d'aménagement forestier, chaque réserve faunique fasse l'objectif, sur la totalité ou une partie de son territoire, d'une gestion intégrée des ressources. »

"Recommandation 7: Que le MRN profite de la mise à jour du régime forestier pour bonifier le processus actuel d'information et de consultation des plans d'aménagement, afin de favoriser la concertation des multiples utilisateurs du milieu forestier. Cette bonification permettra d'assurer une meilleure intégration des besoins et objectifs des principaux utilisateurs lors de la confection des plans d'aménagement forestier $»$.

«Recommandation 9 : Qu'en 2001, le MRN et le MEF rendent compte des progrès accomplis dans l'application de la gestion intégrée des ressources et du milieu forestier et publient à cet effet un bilan. Ce bilan sera intégré aux rapports sur l'état des forêts québécoises et l'état de l'environnement publiés respectivement par le MRN et le MEF.»

Il faut rappeler que les projets-pilotes ont mobilisé beaucoup d'acteurs qui ont travaillé ensemble à mettre sur pied une démarche fonctionnelle de GIR. Ainsi, les acteurs ont beaucoup d'attentes par rapport à cette nouvelle démarche qui permettra, selon eux, d'intégrer dans la planification forestière des objectifs d'aménagement qui prennent en compte les multiples besoins, attentes et valeurs qui se retrouvent sur le territoire forestier québécois. Les nombreuses recommandations qui sont faites par le ministère des Ressources naturelles laissent espérer aux gestionnaires de territoires fauniques structurés (Réserves fauniques, pourvoiries et zecs) que le système prendra enfin en compte leurs préoccupations. Pourtant le seul résultat concret que le MRN produira à la suite de cette fastidieuse démarche sera la production de deux guides qui s'intitulent Planifier la gestion intégrée des ressources et du milieu forestier, où l'un présente la démarche et l'autre présente les méthodes.
Aucune suite tangible n'est proposée après la réalisation des projets-pilotes qui ont mobilisé des acteurs pendant plusieurs années. L'atmosphère de l'époque est empreinte de frustration, de démobilisation et de désengagement par rapport au gouvernement. L'échec de la démarche GIR des années 90 est le déclencheur d'un ras-le-bol collectif à l'endroit du régime forestier québécois, des industriels et du MRN. La crise sociale se matérialisera ainsi dans le documentaire L'Erreur boréale de Richard Desjardins et Robert Monderie qui dévoile au grand public les enjeux de la forêt québécoise. De nombreux acteurs du milieu, dont les communautés autochtones, se joignent à Monderie et Desjardins pour faire entendre leurs désaccords par rapport à l'exploitation des ressources forestières. Il s'agit d'un moment historique au Québec puisque c'est la première fois que les revendications du public viendront ébranler le système forestier. En 2002, la Vérificatrice générale du Québec observe plusieurs irrégularités en lien avec le territoire forestier (Vérificateur général du Québec 2002). Premièrement, le ministère ne détient pas les connaissances suffisantes pour faire un calcul de possibilité forestière. Deuxièmement, le ministère n'a pas les informations requises pour faire un portrait adéquat des ressources forestières.

À la suite des nombreuses questions qui sont soulevées par la population, le gouvernement met sur pied, en 2003, la Commission Coulombe qui a le mandat de lever le voile sur les préoccupations énoncées. Reconnaissant le bien fondé des critiques, la Commission Coulombe recommande de nombreux changements dans la façon de gérer les ressources de la forêt pour atteindre des objectifs d'aménagement durable des forêts. Les intervenants à la Commission sont d'avis qu'il est temps de mettre en œuvre la gestion intégrée telle que le présente l'extrait suivant du rapport de la Commission :

" Les critères de développement durable invitent fortement à adopter une approche globale et intégrée de la gestion du milieu forestier. De nombreux intervenants qui ont participé aux travaux de la Commission ont rappelé cette nécessité. ${ }^{2}$ Dans la pratique, cependant, la gestion actuelle continue d'être centrée sur la récolte de la matière ligneuse pour approvisionner les usines, l'approche tend plutôt d'atténuer les - dommages collatéraux - causés aux autres ressources du milieu et aux autres secteurs d'activités » (Coulombe et al. 2004, p. 204)

Ainsi, la commission propose que les massifs forestiers québécois soient aménagés selon une approche de gestion intégrée et régionalisée. Pour ce faire, il est recommandé de mettre sur pied des commissions régionales sur les ressources naturelles et le territoire (CRRNT) et de faire des plans régionaux de développement intégré des ressources et du territoire (PRDIRT). La Commission recommande aussi que des structures soient mises en place afin d'aider le ministère dans l'élaboration d'un plan d'aménagement forestier

\footnotetext{
$2_{\text {« Outre les groupes de recherche, les groupes environnementaux et }}$ l'Ordre des ingénieurs forestiers du Québec, cette approche est notamment défendue par des industriels, tels que Abitibi Consolidated, surtout présente au Saguenay » (Coulombe et al. 2004, p. 231)
} 
intégré des ressources forestières3. Bien que les CRRNT n'aient pas survécu aux changements de gouvernement et, par le fait même, que les PRDIRT ne soient plus produits, la recommandation 7.5 de la Commission invite le gouvernement à mettre sur pied, dans chaque unité d'aménagement forestier (UAF), des instances responsables de produire des plans d'aménagement forestier intégré et qui seront soumis à des consultations publiques.

En mars 2005, afin de répondre aux recommandations de la Commission Coulombe et d'assurer une utilisation polyvalente et intégrée de la forêt, le ministre des Ressources naturelles et de la Faune propose onze objectifs de protection et de mise en valeur (OPMV) des ressources du milieu forestier qui devront être pris en compte dans les plans généraux d'aménagement forestier de 2008 à 2013. Ces objectifs ont une portée légale et les bénéficiaires de contrats d'aménagement et d'approvisionnement forestiers (CAAF) et de conventions d'aménagement forestier (CvAF) doivent les prendre en compte dans leur planification forestière et, de surcroit, s'engager à les atteindre. Par rapport à l'aménagement intégré, l'un des objectifs vise directement la prise en compte des autres acteurs et il s'intitule Favoriser l'harmonisation des usages en forêt par la conclusion d'ententes écrites consignées au plan général d'aménagement forestier, aussi connu sous le nom d'OPMV-Harmonisation des usages. Cet OPMV oblige les industriels à faire des ententes d'harmonisation avec les autres utilisateurs de la forêt. Plus précisément, quatre étapes doivent être complétées pour répondre aux exigences d'harmonisation des usages :

1. la constitution d'ententes écrites d'harmonisation des usages en matière d'aménagement forestier;

2. le développement de mesures d'harmonisation découlant de ces ententes. Les mesures d'harmonisation constituent les scénarios d'intervention qui devront être appliqués pour respecter les ententes;

3. la cartographie des mesures d'harmonisation, c'est-à-dire, la localisation des superficies touchées par le programme quinquennal où seront appliquées les mesures d'harmonisation;

4. la mise en œuvre et le suivi annuel des mesures d'harmonisation prévues au programme quinquennal. » (Pâquet et Deschênes 2005)

Les réflexions, les échanges et les constats qui ont suivi la Commission Coulombe ont permis de mettre en œuvre un nouveau régime forestier en adoptant en 2013 la Loi sur l'aménagement durable du territoire forestier (LADTF). Notons que l'aménagement intégré est maintenant au cœur de régime forestier puisqu'il apparaît dans le premier article de la loi :

" Article 1.2 : La présente loi institue un régime forestier visant à assurer une gestion des ressources et du territoire qui soit intégrée, régionalisée et axée sur la formulation d'objectifs clairs et cohérents, sur l'atteinte de résultats mesurables et sur la responsabilisation des gestionnaires et des utilisateurs du territoire forestier. » (Éditeur officiel du Québec 2020a)

${ }^{3}$ Recommandation 7.5 de la Commission Coulombe (p. 211)
Ce nouveau régime amène plusieurs changements idéologiques par rapport aux régimes précédents en ce qui concerne la gouvernance des forêts publiques : de nouveaux concepts sont introduits tels que l'aménagement écosystémique; de nouvelles expertises sont requises pour répondre à des objectifs d'aménagement concrets telles que des économistes, des biologistes et des acteurs locaux; de nouvelles connaissances sont nécessaires pour mettre en œuvre les nouveaux concepts et une nouvelle vision doit être mise de l'avant afin d'avoir une perspective globale et plus inclusive (Amedzro St-Hilaire 2013). De façon plus précise, le gouvernement prend la charge de la planification forestière et une plus grande place est accordée aux autres acteurs de la forêt. À ce propos, des Tables locales de gestion intégrée des ressources et du territoire (TLGIRT) sont constituées afin de mieux prendre en compte les valeurs, les besoins et les attentes des différents utilisateurs de la forêt. À cet égard, l'article 55 de la LADTF prescrit la composition des tables tout en donnant aux régions la liberté d'ajouter des postes au sein de leur TLGIRT pour une meilleure représentation de la réalité locale. Plus précisément, le mandat des TLGIRT est de faire des recommandations au ministère pour une meilleure prise en compte des intérêts du milieu. Par contre, les mesures d'harmonisation qui avaient été élaborées grâce aux OPMV ne sont plus valables dans le nouveau régime. Certains acteurs, dont les représentants des territoires fauniques structurés, considèrent qu'il s'agit d'un recul. Depuis 2013, plusieurs ajustements ont été faits au sein des TLGIRT dans le but de mieux définir les rôles et mandats des acteurs impliqués à la table. Les TLGIRT représentent donc le premier outil de consultation publique inscrit dans la réglementation forestière québécoise. Cependant, elles ont été beaucoup critiquées car la gestion intégrée des ressources exige une planification collective de l'ensemble des ressources et à ce jour la possibilité forestière demeure le principal frein à une mise en œuvre efficace de la GIRT. En janvier 2020, on comptait 34 TLGIRT réparties dans toutes les régions administratives du Québec.

Le récent plan stratégique du ministère des Forêts, de la Faune et des Parcs pour les années 2019 à 2023 ne fixe toujours pas d'indicateurs de performance pour évaluer à la mise en œuvre de la GIRT : les indicateurs sont surtout axés sur la performance de l'industrie forestière et un seul indicateur évalue la satisfaction des acteurs par rapport aux mécanismes de consultation (MFFP 2019). Cela dit, il y a un écart entre la satisfaction des acteurs et la mise en ouvre de la GIRT puisque la satisfaction par rapport aux approches de consultation peut être positive sans toutefois que la GIRT ne soit mise en pratique.

Bien que la réglementation ait été bonifiée pour mieux répondre aux besoins de la société, nous croyons qu'il est nécessaire d'être prudent dans l'analyse des résultats surtout en ce qui concerne les engagements pris vis-à-vis les acteurs. Au fil du temps, la GIRT s'est complexifiée en incluant de nombreux concepts qui, concrètement, ne sont pas pris en compte ni mesurés dans un contexte d'aménagement forestier au Québec. Suite à l'analyse du concept tel que proposé par Desrosiers et al. (2010), nos travaux nous incitent à proposer une définition plus réaliste et mesurable (Bernard et Gélinas, 2020) qui clarifie le terme GIRT afin de mieux 
répondre à la réalité de la gestion intégrée en forêt québécoise. Nous proposons la notion de " gestion intégrée des ressources forestières » (GIRF) qui pourrait se définir ainsi : « La GIRF est un processus de consultation régionale entre les parties prenantes impliquées sur le territoire forestier. Ce processus continu vise à intégrer les enjeux en amont, et ce, tout au long du processus. La GIRF vise l'atteinte d'une équité sociale entre les parties prenantes en considérant leurs besoins et leurs attentes »(Bernard et Gélinas, 2020).

\section{Changements observés de 1986 et à d'aujourd'hui}

Cette section présente les principaux changements observés au cours des trente dernières années en ce qui concerne la mise en œuvre du concept de GIR. Pour produire ce constat, nous nous sommes basés sur les propos des experts ayant participé au colloque de 2018 ainsi que sur les actes du colloque de 1986. Afin d'aider les experts dans leur présentation, le résumé de l'acte du colloque de 1986 a été soumis à tous les participants avant la tenue du colloque. Les résultats exposés ici sont issus des présentations faites au colloque et aux échanges qui en ont suivi.

Premièrement, à l'époque, les communautés autochtones n'étaient pas prises en compte dans l'utilisation polyvalente de la forêt. Bien que revendiquée depuis plusieurs années, la place de ces communautés dans la gestion des ressources naturelles est maintenant reconnue et des dispositions particulières ont été adoptées pour faciliter les négociations avec elles. Par exemple, des consultations réservées aux communautés autochtones sont organisées par le gouvernement du Québec pour leur présenter les plans d'aménagement intégré tactique et opérationnel (PAFIT et PAFIO). Certes, les négociations sur les revendications territoriales des Premières Nations sont toujours en cours, mais la volonté de considérer les communautés autochtones dans la gestion du territoire forestier est de plus en plus marquée (Curran et Gonigle 1999; Stevenson et Perreault 2008; Maclean et al. 2015).

Dans un deuxième temps, il est maintenant question d'aménager les forêts selon des principes d'aménagement écosystémique visant à assurer le maintien de la biodiversité et la viabilité des écosystèmes tout en diminuant les écarts entre la forêt aménagée et la forêt naturelle (en s'inspirant, notamment du régime de perturbations naturelles) (Gauthier et al. 2008). Il y a trente ans, les interventions forestières étaient plutôt centrées sur l'approvisionnement de l'industrie forestière et, dans la mesure du possible, on visait une atténuation des impacts de la coupe sur les écosystèmes forestiers. Au fil des décennies, plusieurs chercheurs se sont intéressés à ces nouvelles approches d'aménagement (Attiwill 1994; Bergeron et Harvey 1997; Franklin 1997). De plus, pour encadrer les pratiques forestières, le règlement des normes d'intervention (RNI) a été sanctionné par le gouvernement en 1988. En 2018, le RNI a été remplacé par le règlement sur l'aménagement durable des forêts (RADF) pour répondre aux exigences de la LADTF.

Troisièmement, à l'époque, aucune structure officielle ne permettait aux acteurs présents sur le territoire d'échanger avec le gouvernement. Le ministère obligeait les industriels à tenir des consultations publiques sur leurs plans d'aménagement afin de rendre le processus plus transparent (Amedzro St-Hilaire 2013). Les consultations publiques se faisaient sur des plans d'aménagement qui étaient mis en œuvre par les industriels. Il était ainsi difficile pour les parties prenantes de proposer des modifications à la fin du processus de planification. Cela dit, en fonction des revendications et de leurs impacts sur les opérations forestières, les industriels faisaient des ententes à la pièce avec les autres acteurs qui insistaient pour être dédommagés. Ce processus d'harmonisation était donc fait sur une base continue sans partage d'informations entre les acteurs. Ces rencontres se faisaient sur une base ponctuelle et majoritairement en lien avec des enjeux de nature opérationnelle. Bref, les industriels forestiers avaient le beau jeu, celui de négocier à la pièce avec les acteurs. C'est pourquoi la Commission Coulombe concluait que l'État devait reprendre le contrôle de l'aménagement des forêts publiques. À la suite de l'entrée en vigueur de la LADTF, certains outils de mise en œuvre de la GIRT ont été adoptés tels que les TLGIRT. Ces structures facilitent la communication entre les acteurs et surtout, ceux-ci sont en mesure de mieux comprendre les préoccupations des autres acteurs présents aux tables. Selon la loi, ces tables ont le mandat de faire des recommandations stratégiques aux professionnels du ministère de la Forêt, de la Faune et des Parcs pour qu'ils intègrent les valeurs sociales dans le plan d'aménagement forestier intégré. Aussi, au fil du temps, on note que la perception des multiples utilisateurs de la forêt a évolué. À l'époque du colloque de 1986, hormis les industriels, les autres acteurs devaient défendre leur légitimité à occuper le territoire. Aujourd'hui, leur légitimité est reconnue. Ils revendiquent maintenant la prise en compte de leurs valeurs et de leurs besoins dans l'aménagement forestier.

Quatrièmement, la place de la conservation est beaucoup plus importante aujourd'hui qu'elle ne l'était à l'époque. Par exemple, les aires protégées font maintenant partie du paysage forestier québécois et leur nombre d'aires protégées a grandement augmenté. À ce propos, en 1987, le registre des aires protégées du Québec tenait son premier bilan de la superficie des aires protégées, évaluée à $0,36 \%$ de la province tandis qu'en 2020, ce même registre compte $10,04 \%$ du territoire québécois en aires protégées (MELCC 2020). Le gouvernement du Québec reconnaît l'importance de ces territoires. Actuellement, l'enjeu est plutôt de bonifier et de consolider le réseau d'aires protégées dans le sud du Québec puisque l'ajout de superficie en aire protégée réduit, entre autres, la possibilité forestière. Plusieurs avancées ont été observées depuis les trente dernières années, mais le Québec est encore loin de rencontrer ses engagements pris en 2015 celui d'atteindre la cible internationale de $17 \%$ d'aires protégées d'ici 2020.

Cinquièmement, plusieurs pratiques ont été éradiquées au cours des 30 dernières années. Par exemple, le flottage du bois n'est plus permis au Québec depuis 1995. Sous la pression de nombreux groupes écologistes, cette pratique a été abandonnée pour laisser uniquement la place au transport par camion ou par voie ferroviaire. À ce propos, en 1986, une des conférences portait sur les impacts négatifs de cette pratique sur les milieux aquatiques et riverains. À cette époque, douze rivières étaient toujours utilisées pour le flottage. Notons aussi que l'utilisation de pesticides chimiques a été abolie au Québec en 2001. La lutte biologique et l'application 
d'une sylviculture préventive sont aujourd'hui les principaux outils pour répondre aux attaques des insectes ravageurs. De plus, les pratiques forestières dans les milieux riverains et humides ont beaucoup changé et sont mieux réglementées. Le règlement d'aménagement durable des forêts encadre maintenant de manière plus stricte les traverses de cours d'eau comme l'indiquent les articles 99 à 108 dudit règlement (Éditeur officiel du Québec 2020b).

Finalement, le dernier constat que nous faisons est en lien avec la certification forestière puisqu'elle fait maintenant partie intégrante de la foresterie québécoise. Dans les années 80 , la certification ne faisait pas partie du portrait forestier. À l'heure actuelle, au Québec, on compte trois systèmes de certification soit le Forest Stewardship Council (FSC) fondé en 1994, le système Sustainable Forest Initiative (SFI) fondé en 1995 et le système de la Canadian Standard Association (CSA) développé en 1996. L'importance de la certification forestière est le résultat des discussions et des recommandations faites lors du Sommet de la Terre en 1992 à Rio. L'objectif principal de ces certifications est d'encourager les entreprises à adopter des pratiques plus respectueuses de l'environnement et ainsi assurer aux consommateurs que les produits qu'ils achètent sont produits dans un contexte d'aménagement durable. Aujourd'hui, près de $90 \%$ des forêts publiques québécoises sont certifiées selon l'un des trois systèmes de certification (MFFP 2020b).

\section{Perspectives des experts responsables de la mise en œuvre de la GIRT}

La section qui suit présente des résultats de recherche qui ont été collectés au cours d'entrevues semi-dirigées faites auprès de 31 experts impliqués dans la mise en ouvre de la GIRT. Ces résultats issus d'une étude plus exhaustive (Bernard et Gélinas, 2020) présentent l'analyse des réponses des experts à la question suivante : « Par rapport à l'ancien régime, croyezvous que le nouveau régime facilite la mise en ouvre de la GIRT? ».

La majorité des répondants ont souligné que le régime forestier de 2013 est un pas de plus vers la mise en œuvre de la GIRT puisqu'on accorde une plus grande place à la consultation des acteurs régionaux et du public. Le régime forestier de 2013 est qualifié comme étant plus inclusif. Cependant, la plupart des répondants soulignent aussi que le nouveau régime est beaucoup plus complexe et qu'il alourdit les échanges avec les acteurs. Plusieurs mentionnent qu'auparavant il était plus simple pour les acteurs de communiquer avec les responsables des opérations forestières. Pour certains, la rupture du canal de communication peut nuire à la cohabitation sur le territoire. En effet, lorsque les acteurs sont insatisfaits des interventions, ils ne savent plus nécessairement à qui s'adresser. Pour illustrer cet enjeu, plusieurs experts ont donné l'exemple du réseau routier qui est l'un des éléments les plus importants en ce qui concerne l'utilisation multiple du territoire forestier. À l'époque, les utilisateurs pouvaient s'adresser directement aux responsables des opérations pour s'entendre sur l'entretien ou la mise en place du réseau routier. Aujourd'hui, puisque le ministère doit valider l'ensemble des interventions, il devient plus difficile d'avoir des ententes informelles entre les acteurs et les responsables des opérations. Tout doit passer par un canal de communication officiel, laissant ainsi moins de flexibilité pour faire de petits ajustements directement en forêt. Les deux extraits qui suivent illustrent cette rupture communicationnelle entre l'ancien et le nouveau régime:

"C'est des relations qui étaient établies entre les industriels et les gens du milieu qui se connaissaient [...]. Il y a un lien qui est brisé qui crée plus de frustrations.» (E14).

« [...] Pour certains tiers, ils diraient que c'est plus compliqué maintenant. Avant j'appelais le gars du moulin :

- Peux-tu m’arranger mon chemin, il est magané.

- Ah oui, pas de problème, je vais t'arranger ça!»

Pis là ça se réglait comme ça. Tandis que maintenant, je parle à qui? Si on a juste le canal de la TLGIRT. [...] Dans les premières années du régime, ils s'adressaient aux industriels, comme il le faisait avant. » (E27)

La modification de la législation forestière amène aussi un changement de mentalité quant à l'aménagement des ressources forestières. Un des experts souligne que dans le nouveau régime on se base sur une échelle stratégique plutôt qu'opérationnelle, ce qui est aussi une nouveauté de la nouvelle réglementation. Certes auparavant, les ententes se faisaient plus rapidement, mais la démarche devait être reprise pour chaque nouveau secteur de coupe. Aujourd'hui, le régime tente d'inclure des aspects stratégiques qui réduisent les requêtes individuelles pour prendre des décisions stratégiques qui répondent mieux à un ensemble de préoccupations d'aménagement du territoire.

"Ce que j'entends souvent des gens autour de la table [TLGIRT], pis des BGA [bénéficiaire de garantie d'approvisionnement], c'est que ça allait mieux avant. Ils s'entendaient sur le houde du pick-up. Ils s'entendaient pour celui qui levait la main et qui criait. [...] Ce n'était pas stratégique. [...] C'était vraiment du un à un, opérationnel. Il n'y avait pas d'intégration. » (E31)

Il y a donc deux visions par rapport à la mise en ouvre de la GIRT soit une vision sur une plus longue durée (stratégique) et une vision de courte durée (opérationnelle). On comprend aussi que certains acteurs préfèrent les décisions de longue durée en raison de la qualité stratégique de ces décisions pour la collectivité plutôt que les ententes à la pièce qui se font souvent entre un nombre restreint d'acteurs.

Dans le nouveau régime forestier, la planification forestière est réservée aux fonctionnaires de l'État. Néanmoins, le fait que l'État soit responsable de la planification n'est pas perçu négativement par les experts. Un des répondants mentionne que la prise en charge de la gestion forestière par le gouvernement plutôt que par l'industrie facilite l'établissement d'un climat de confiance. Un autre répondant mentionne aussi l'imputabilité du gouvernement envers la population québécoise, imputabilité que n'avait pas l'industrie forestière lorsqu'elle était responsable de planifier la récolte forestière.

« Reste que le ministère dans le régime actuel a une forme d'imputabilité et puis il doit rendre des comptes au public et aux autorités par rapport à ce qu'il fait. [... ] on est obligé de faire ce qu'on dit et on est obligé de le 
démontrer. L'industrie n'avait pas ces comptes-là à rendre au public. » (E7)

Cependant, certains répondants critiquent le manque de recul du ministère dans le nouveau régime en ayant un double mandat soit celui de vérifier l'application de la loi et celui d'assurer une garantie d'approvisionnement aux industriels.

«[...] le livre vert prévoyait un organisme indépendant des entreprises et du gouvernement pour faire l'aménagement forestier. [...] Pour que les industriels se confinent dans leur rôle d'industriel et que le gouvernement se confine dans son rôle de vérificateur et d'applicateur de législation. Aujourd'hui, en faisant ça [en parlant du rôle de vérificateur et d'applicateur de législation] et la planification, je trouve qu'il manque de recul. Pas qu'ils le font mal, mais je pense que ça nuit à la confiance du public. [...] J'aurais aimé ça qu'on essaie la séparation complète. » (E25)

Un autre répondant ajoute que le ministère n'est plus aussi neutre qu'il ne l'était par le passé lorsqu'il jouait un rôle de surveillance : «Avant le ministère avait plus de recul, il était plutôt un juge. Là, c'est plus à l'intérieur que ça devient politique. [...] Parfois ce serait peut-être mieux d'avoir du recul. » (E14).

La complexité du nouveau régime n'est pas uniquement vue de manière négative. Cette complexité est aussi associée à la capacité du ministère de pouvoir approfondir des questions en lien avec les nouvelles pratiques forestières, capacité qui, selon les répondants, n'était pas aussi grande lorsque l'industrie était responsable de la planification des récoltes. À ce propos un des répondants explique ce phénomène :

« Les exigences territoriales, les exigences d'harmonisation se sont multipliées par dix. Je suis toujours surpris de la quantité d'informations qu'on doit gérer, au niveau des harmonisations, au niveau de la planification, au niveau du respect des entités territoriales. Je te dirais qu'une des bonnes choses que je vois, c'est que c'est le ministère qui est responsable de la planif [planification forestière]. Je ne verrais pas ça dans les mains du privé. [...] L'industrie n'avait pas les moyens d'aller dans un niveau d'aménagement aussi complexe que ça. Là, on est rendu dans la foresterie écologique, écosystémique. »(E3)

Dans certaines régions, les membres des TLGIRT s'appuient sur les professionnels du ministère pour répondre aux enjeux qui sont soulevés lors des rencontres de la TLGIRT. Il faut rappeler qu'à la base, la mission de la TLGIRT est de proposer des recommandations aux professionnels du ministère pour intégrer des enjeux stratégiques dans la planification forestière. Ainsi, que les membres des TLGIRT demandent aux professionnels du gouvernement de répondre à leur questionnement est contradictoire avec la mission des TLGIRT qui doivent agir indépendamment. L'extrait qui suit présente cette situation et permet de mieux saisir la position des professionnels du gouvernement : " Aux yeux de certains membres de la TLGIRT, on est devenu le bras exécutant de la TLGIRT. La TLGIRT veut quelque chose, alors le ministère doit le faire. Alors que dans l'esprit de la Loi, la TLGIRT est là pour alimenter le ministère pour qu'on intègre certaines valeurs dans le PAFI »(E7). Les rôles des membres des TLGIRT sont clairement définis dans le guide des TLGIRT (Bernier et Le Goff 2018). À la lumière de ce témoignage, dans certaines régions, les représentants du ministère jouent un double rôle soit celui de représenter le ministère et celui d'experts ou de consultants. On peut toutefois voir cet enjeu comme étant un beau problème. Si les participants aux TLGIRT sollicitent les représentants du gouvernement, c'est que d'une part, ils leur font confiance, et que d'autre part ils désirent améliorer leurs connaissances par rapport aux enjeux territoriaux. Néanmoins la division des rôles peut aussi devenir contraignante et limiter l'avancement des travaux d'une TLGIRT. Nous pouvons penser que dans certaines régions les experts supportent plusieurs régions. Il ne faudrait donc pas limiter les travaux pour des contraintes administratives. Les TLGIRT devraient être centrales au processus d'élaboration des plans d'aménagement forestier intégré, même s'il peut s'agir d'une idée complexe à mettre en œuvre en raison de la mobilisation variable des membres des TLGIRT (Bernard and Gélinas, 2020).

\section{Conclusion et recommandations}

À la lumière des sections précédentes, nous sommes en mesure d'élaborer des recommandations et des constats par rapport à la mise en ouvre de la gestion intégrée dans le contexte forestier québécois.

\section{Gouvernance et leadership de la gestion intégrée}

Le gouvernement via la LADTF a le mandat d'aménager les forêts québécoises selon un aménagement intégré. Cela dit, l'aménagement intégré est un concept beaucoup plus social que forestier, d'où l'importance de décentraliser les pouvoirs. La décentralisation est déjà en cours et de plus en plus d'outils de participation publique ont été développés au fil du temps (Mcgurk et al. 2006; Chiasson et Leclerc 2013; Tardif et al. 2017). Cependant, pour proposer un plan d'aménagement intégré qui reçoit une réelle approbation sociale, il faut que ce dernier ait reçu la licence sociale à opérer. Le concept de la licence sociale à opérer est très présent lorsqu'il est question de projet d'exploitation des ressources naturelles et Black (2013, p. 18) la définit comme suit :

«The social license to operate is not a piece of paper or a document like a government license. It's a form of social acceptance or approval that companies or projects earn through consistent and trustworthy behaviour and interactions with their stakeholders. It's a socially constructed perception that your company or project has a legitimate place in that community. "

D'autres concepts sont associés à la licence sociale à opérer tels que la légitimité, la confiance, le consentement, la notion de bénéfices, la participation et la transparence (Morrison 2014). Bref, autant de concepts qui font écho à l'aménagement des forêts publiques québécoises et à la gestion intégrée des ressources forestières. Les projets-pilotes des années 90 avaient permis de développer une démarche pour obtenir cette licence sociale puisque les acteurs devaient formuler leurs objectifs en lien avec l'utilisation du territoire. Une fois ces objectifs énoncés et connus des autres parties, ils devaient être pris en considération dans l'élaboration de la planification. Bref, cette approche permettait de mettre en place des interventions forestières légitimes, consenties et transparentes. Cela dit, pour 
mettre en œuvre une telle approche, il faut qu'un leadership clair et précis soit établi, et ce, au-delà des recommandations faites par les représentants siégeant aux tables de gestion intégrée. Les recommandations des membres des TLGIRT sont valables et légitimes, mais elles ne sont pas suffisantes pour octroyer au gouvernement une licence sociale à opérer. Ultimement, la réalisation du plan d'aménagement revient aux professionnels du ministère qui prennent ou non en compte les recommandations des membres des TLGIRT. Dans un monde idéal, il faudrait que les personnes responsables d'élaborer les plans d'aménagement forestier soient autonomes, sans dépendre de la possibilité forestière. Ils devraient plutôt être en mesure d'influencer la possibilité forestière afin d'inclure les autres ressources et d'éliminer du jargon forestier les activités et les ressources contraignantes. Aucune activité ou ressource ne devrait être vue comme des contraintes, mais plutôt comme des opportunités. Il faudrait aussi que les plans d'aménagement intégré prennent en compte les objectifs régionaux des acteurs. L'entité responsable de la planification forestière devrait avoir toutes les informations nécessaires pour assurer une utilisation optimale du territoire forestier tout en répondant aux objectifs des parties prenantes. Il faudrait aussi inclure le concept d'imputabilité pour assurer une saine gestion. Pour ce faire, une structure neutre devrait être nommé pour s'assurer que les responsables de la planification répondent aux objectifs de la stratégie d'aménagement durable des forêts. De cette manière, l'aménagement forestier serait plus acceptable et il serait possible de redonner une place aux professionnels de la forêt afin de prendre des décisions plus éclairées qui visent le bien commun. À l'heure actuelle, le ministère des Forêts, de la Faune et des Parcs est juge et parti en ce qui concerne la récolte forestière. D’une part, il doit assurer l'approvisionnement des usines et d'autre part il doit répondre aux requêtes des acteurs sur le territoire. À ce propos, il faut noter que dans certaines régions le nombre d'acteurs impliqués sur le territoire forestier a grandement augmenté et la récolte forestière devient une contrainte importante. Une clarification du leadership et des responsabilités en lien avec l'aménagement des ressources forestières faciliterait certainement l'atteinte des objectifs d'aménagement intégré.

La complexité du processus d'aménagement est certainement une des contraintes à la GIRT. Un grand nombre d'acteurs sont impliqués dans le processus d'aménagement forestier et il n'est pas toujours clair de comprendre tous les enjeux d'aménagement. Ainsi, si les informations étaient centralisées à un endroit, il serait plus simple d'identifier les forces, faiblesses, opportunités et menaces pour la mise en œuvre de la GIRT, et ce, dans un contexte régional. Pour répondre à cet enjeu, nous proposons que la production de chaque plan d'aménagement forestier intégré soit faite par un gestionnaire unique. Le concept de gestionnaire unique ne signifie pas qu'une seule personne ait la responsabilité de produire le plan; le gestionnaire unique pourrait prendre la forme d'une équipe multidisciplinaire adaptée au contexte régional. À l'heure actuelle, on considère que le territoire forestier québécois est aménagé par plusieurs gestionnaires, soit les professionnels du milieu forestier, les professionnels du milieu faunique et les professionnels du milieu récréatif. Le manque de cohésion entre les gestionnaires explique en partie la complexité du processus d'aménagement forestier.
Comme proposé par la Commission Coulombe, des commissions régionales sur les ressources naturelles (CRRNT) ont été mises sur pied pour assurer la centralisation de l'aménagement intégré. Ces structures ont toutefois été abolies en 2015 suite à l'adoption du projet de loi $n^{\circ} 28^{4}$ laissant ainsi une période de latence pendant laquelle les acteurs et les parties prenantes se sont démobilisés par rapport à la mise en œuvre de la GIRT dans leurs régions (Tardif et al. 2017). À l'époque, la CRRNT avait le leadership nécessaire pour regrouper l'ensemble des acteurs. De plus, cette instance était un acteur neutre par rapport aux questions forestières. Bref, il faut tenter de retrouver cet équilibre dans l'écosystème forestier.

\section{Vision territoriale de la gestion intégrée}

Les différentes parties prenantes impliquées sur le territoire forestier travaillent à différentes échelles et les mésententes sont souvent causées par ce décalage. Par exemple, le ministère tente d'établir des objectifs d'aménagement qui répondront à des enjeux stratégiques pour la région. Cependant, les acteurs sur le territoire sont souvent préoccupés par des enjeux à plus petite échelle. Avant d'entamer des réflexions collectives, il faudrait comprendre les attentes des parties prenantes aux TLGIRT par rapport à leur participation. Cet exercice permettrait ainsi de mieux saisir l'échelle territoriale qui préoccupe les acteurs.

Les entrevues semi-dirigées ont aussi permis de soulever une nouvelle question par rapport à la gestion intégrée, soit celle de la différenciation entre l'harmonisation par rapport à l'intégration des enjeux soulevés par la TLGIRT. De prime abord, l'harmonisation semble faire référence aux ententes ciblées entre deux ou plusieurs acteurs pour répondre à un enjeu très précis. L'intégration telle qu'on l'entend dans GIRT fait plutôt référence à une prise en compte globale d'une ou plusieurs préoccupations partagées par un ou plusieurs acteurs qui pourra se matérialiser dans une orientation d'aménagement forestier, et ce, dans une perspective à long terme.

\section{Flexibilité du système forestier}

Le manque de flexibilité du système forestier peut s'expliquer par la primauté de la possibilité forestière sur les autres ressources (Bernard and Gélinas, 2020). Cela dit, la gestion intégrée ne vise pas à éradiquer la coupe de bois. Au contraire, elle vise à perpétuer les pratiques forestières tout en incluant d'autres activités. En centralisant la planification forestière, le gouvernement a évacué de l'équation tous les acteurs qui pourraient contribuer à la réalisation d'un plan d'aménagement forestier intégré. Aux États-Unis, plusieurs professionnels sont impliqués dans l'élaboration du plan d'aménagement forestier. Le Québec ne pourrait-il pas s'inspirer de cette approche pour revoir ses pratiques de planification forestière tout en mettant à profit les structures de participation publique?

\footnotetext{
${ }^{4}$ Projet de loi $\mathrm{n}^{\circ} 28$ s'intitulait Loi concernant principalement la mise en œuvre de certaines dispositions du discours sur le budget $d u$ 4 juin 2014 et visant le retour à l'équilibre budgétaire en 2015-2016
} 
Dans un contexte où l'acceptabilité sociale est au cœur des décisions politiques (Batellier et Maillé 2017), l'utilisation d'approches plus inclusives et moins complexes permettrait certainement de faciliter les synergies entre les acteurs tout en répondant aux enjeux d'aménagement durable des forêts.

\section{Mettre en place un projet de société 2.0}

Au fil des décennies, le milieu forestier québécois a connu de nombreux changements idéologiques. La nationalisation des ressources naturelles, la réappropriation du territoire forestier par le gouvernement du Québec et la prise en compte des différentes parties prenantes dans la mise en œuvre de l'aménagement des forêts en sont quelques exemples. Bien que le système forestier se soit adapté pour mieux répondre aux enjeux sociaux et environnementaux, le nombre d'acteurs présents sur le territoire forestier a crû au cours des dernières années tout comme les préoccupations en lien à l'aménagement et la conservation des forêts. Pour réduire les conflits d'usage, il serait important de bonifier les plans d'affectation des terres publiques (PATP). À ce jour, le territoire forestier est vu comme un bloc uniforme. La mise à jour du PATP permettrait ainsi d'impliquer les parties prenantes pour définir des affectations multifonctionnelles ou au contraire des affectations pour des activités spécifiques. Un autre élément qui devrait être pris en compte, et ce, depuis plusieurs décennies, est la mise en œuvre d'approches régionales de forêts communautaires. À ce propos, différents projets ont été proposés par le gouvernement tel que les forêts habitées et les forêts de proximité. Ceci dit, aucun modèle n'a réellement été mis en œuvre dans les régions du Québec. De nombreuses démonstrations ont été faites pour présenter les avantages de modèles régionaux d'aménagement des ressources forestières (Guertin et Bouthillier 1996, Gélinas et Bouthillier, 2005; Teitelbaum 2013). Finalement, le projet de société 2.0 en lien avec l'aménagement du territoire forestier devra s'intéresser aux différentes cultures forestières qui existent au Québec (Bernard et Gélinas, 2020). Cette clarification permettra de mieux répondre aux enjeux sociaux qui bloquent l'innovation en lien avec la gouvernance forestière. Lorsque les professionnels du milieu forestier seront en mesure d'appréhender les cultures forestières, des stratégies de communication, de gestion intégrée ou de résolution de problèmes pourront être élaborées. D'ici là, nous devons continuer à évoluer dans un contexte qui oppose la récolte de bois aux autres activités qui ont lieu en forêt. Il faut donc continuer d'étudier les nouvelles approches de gouvernance forestière qui permettent d'améliorer la compréhension des enjeux sociaux en vue d'atteindre les objectifs d'aménagement durable que le gouvernement s'est fixés.

\section{Remerciements}

Nous tenons à remercier tous les participants et participantes au colloque Où en sommes-nous 30 ans après le colloque "L'utilisation polyvalente de la forêt, une utopie? qui s'est tenu le 9 mai 2018 dans le cadre du colloque annuel de l'ACFAS 2018. La réalisation de cet article n'aurait pas été possible sans la contribution des nombreux professionnels, chercheurs et étudiants qui ont collaboré à la réussite de ce colloque. Nous remercions aussi la contribution financière de Génome Québec/Génome Canada [243FOR] et le Fonds de recherche société et culture du Québec dans le cadre du programme de bourse au doctorat.

\section{Références}

Amedzro St-Hilaire, W.G. 2013. Réforme des politiques forestières au Québec et dynamique interne des structures étatiques de gestion forestière, In: Chiasson, G., Leclerc, É. (Eds.), La Gouvernance Locale Des Forêts Publiques Québécoises - Une Avenue de Développement Des Régions Périphériques? Presses de l'Université du Québec, pp. 65-88.

Attiwill, P.M. 1994. The disturbance of forest ecosystems : The ecological basis for conservative management. For. Ecol. Manage. 63: 247-300.

Batellier, P. et M.-È. Maillé 2017. Acceptabilité sociale: sans oui, c'est non, Les Éditio. ed. Montréal, Canada. 301 pp.

Bergeron, Y. et B. Harvey. 1997. Basing silviculture on natural ecosystem dynamics : An approach applied to the southern boreal mixedwood forest of Quebec. For. Ecol. Manage. 92: 235-242.

Bernard, A. et Gélinas, N. 2020. Assessment of a Public Participation Tool by Experts: A Case Study of Quebec, Canada. Natural Resources. 11, 282-306

Bernier, S. et H. Le Goff. 2018. Guide de la table locale de gestion intégrée des ressources et du territoire : son rôle et son apport dans l'élaboration des plans d'aménagement forestier intégré. Ministère des Ressources naturelles, de la Faune et des Parcs, Québec. 31 pp. Black, L. 2013. Social License to Operate : Your Management Framework for Complex Times. Routledge. ed. Taylor \& Frances. 101 pp. Chiasson, G. et É. Leclerc. 2013. La gouvernance locale des forêts publiques québécoises, Les Presse. ed. Québec, Canada. 249 pp.

Coulombe, G., J. Huot, J. Arsenault, É. Bauce, J.-T. Bernard, A. Bouchard, M.-A. Liboiron et G. Szaraz. 2004. Commission d'étude sur la gestion de la forêt publique québécoise. Québec. $304 \mathrm{pp}$.

Curran, D. et M.M. Gonigle. 1999. Aboriginal forestry : Community management as opportunity and imperative. Osgoode Hall Law J. 37: 711-774.

Desrosiers, R., S. Lefebvre, P. Munoz, et J. Pâquet. 2010. Guide sur la gestion intégrée des ressources et du territoire : son application dans l'élaboration des plans d'aménagement forestier intégré. Ministère des Ressources naturelles et da la Faune. 34 pp.

Éditeur officiel du Québec. 2020a. A-18.1 Loi sur l'aménagement durable du territoire forestier. Gouvernement du Québec, Québec, Canada.

Éditeur officiel du Québec 2020b. Règlement sur l'aménagement durable des forêts du domaine de l'État. Gouvernement du Québec, Québec, Canada.

Fédération québécoise des gestionnaires de zecs. 2003. Les zecs, 25 ans d'histoire à retracer. Québec, Canada. 99 pp.

Franklin, J.F. 1997. Ecosystem management: An overview, In: Boyce, M.S., Haney, A. (Eds.), Ecosystem Management: Applications for Sustainable Forest and Wildlife Resources. New Haven, Connecticut, pp. 21-53.

Gauthier, S., M.-A. Vaillancourt, A. Leduc, L. De Grandpré, D. Kneeshaw, H. Morin, P. Drapeau et Y. Bergeron. 2008. Aménagement écosystémique en forêt boréale. Presses de l'Université du Québec. 600 pp.

Gélinas, N. et L. Bouthillier. 2005. La forêt habitée: Un modèle de gestion partenariale? Analyse de la perception des participants à cinq projets au Québec. Cah. Geogr. Que. 49: 157-175.

Guertin, C.-éric et L. Bouthillier. 1996. Les conditions d'établissement des forêts communautaires au Québec. In: Côté, S., Klein, J.L., Proulx, M.-U. (Eds.), Action Collective et Décentralisation. Rimouski, pp. 209-222.

Maclean, K., C.J. Robinson et D.C. Natcher. 2015. Consensus Building or Constructive Conflict ? Aboriginal Discursive Strategies to Enhance Participation in Natural Resource Management in Australia and Canada. Soc. Nat. Resour. 28: 197-211. https:// doi.org/10.1080/08941920.2014.928396 
Mcgurk, B., A.J. Sinclair, A. Diduck, B. Mcgurk, A.J. Sinclair, A. Diduck, A. Assessment et A.J. Sinclair. 2006. An Assessment of Stakeholder Advisory Committees in Forest Management : Case Studies from Manitoba , Canada. Soc. Nat. Resour. 19: 809-826. https://doi.org/10.1080/08941920600835569

MELCC. 2020. Registre des aires protégées [WWW Document]. URL http://www.environnement.gouv.qc.ca/biodiversite/aires_ protegees/registre/ (accessed 2.29.20).

MFFP. 2019. Plan stratégique 2019-2023. Québec, Canada.

MFFP. 2020a. Inventaire écoforestier [WWW Document]. URL https://mffp.gouv.qc.ca/les-forets/inventaire-ecoforestier/ (accessed 3.23.20).

MFFP. 2020b. Certification forestière [WWW Document]. URL https://mffp.gouv.qc.ca/les-forets/amenagement-durable-forets/ certification-forestiere/ (accessed 2.24.20).

Ministère des Ressources naturelles. 1997. Planifier la gestion intégrée des ressources et du milieu forestier - Une démarche. Québec, Canada.

Ministère des Ressources naturelles, Ministère de l'Environnement et de la Faune. 1998. La mise en oeuvre de la gestion intégrée des ressources du milieu forestier : Des recommandations. Québec, Canada.

Morrison, J. 2014. The Social License - How to Keep Your Organization Legitimate. Palgrave Macmillan, London, UK. https:// doi.org/10.1057/9781137370723
Pâquet, J. et L. Deschênes. 2005. Lignes directrices pour la mise en oeuvre des objectifs visant le maintien de la qualité des paysages et l'harmonisation des usages. Québec, Canada.

Regroupement pour un Québec Vert 1988. L'utilisation polyvalente de la forêt: une utopie? In: 54e Congrès Annuel de l'ACFAS. Montréal, Canada, pp. 1-233.

Réseau Zec. 2020. Qu'est-ce qu'une zec? [WWW Document]. URL https://www.reseauzec.com/le-reseau

Sépaq. 2020. Réserves fauniques [WWW Document]. URL https://www.sepaq.com/rf/reseau.dot (accessed 6.12.20)

Stevenson, M.G. et P. Perreault. 2008. Capacity For What ? Capacity For Whom ? Aboriginal Capacity and Canada's Forest Sector. Edmonton, Alberta.

Tardif, J., J.F. Bissonnette et J. Dupras. 2017. La participation publique dans la gestion des forêts du Québec: Réorganisation de la concertation régionale dans un contexte institutionnel en transition. For. Chron. 93: 58-70. https://doi.org/10.5558/tfc2017-011

Teitelbaum, S. 2013. L'arbre est dans ses feuilles et la forêt, dans sa communauté, Guide sur la foresterie communautaire.

Vérificateur générale du Québec. 2002. Rapport à l'Assemblée nationale pour l'année 2001-2002. Québec, Canada. (accessed 3.23.20) 